\title{
Genes, cognition and dyslexia: learning to read the genome
}

\author{
Simon E. Fisher ${ }^{1}$ and Clyde Francks ${ }^{2}$ \\ ${ }^{1}$ Wellcome Trust Centre for Human Genetics, University of Oxford, Roosevelt Drive, Oxford, OX3 7BN, UK \\ ${ }^{2}$ Translational Medicine and Genetics, GlaxoSmithKline, Via Fleming 4, 37100 Verona, Italy
}

\begin{abstract}
Studies of dyslexia provide vital insights into the cognitive architecture underpinning both disordered and normal reading. It is well established that inherited factors contribute to dyslexia susceptibility, but only very recently has evidence emerged to implicate specific candidate genes. In this article, we provide an accessible overview of four prominent examples - DYX1C1, KIAA0319, DCDC2 and ROBO1 - and discuss their relevance for cognition. In each case correlations have been found between genetic variation and reading impairments, but precise risk variants remain elusive. Although none of these genes is specific to readingrelated neuronal circuits, or even to the human brain, they have intriguing roles in neuronal migration or connectivity. Dissection of cognitive mechanisms that subserve reading will ultimately depend on an integrated approach, uniting data from genetic investigations, behavioural studies and neuroimaging.
\end{abstract}

\section{Introduction}

Unlike the virtually effortless and automatic acquisition of spoken language during the first few years of life, learning to read is a challenging task that requires extensive tuition. To become proficient in reading, writing and spelling, a child must develop an explicit awareness of the structural elements of language and of how these relate to an arbitrarily defined set of visual symbols. Moreover, the act of reading places unusual demands on the brain, depending on a high degree of visual acuity, fine motor control, rapid temporal processing and so on. Written language is a cultural innovation that appeared relatively recently in the history of our species; archaeological evidence suggests that sophisticated writing systems emerged just a few thousand years ago [1]. It is therefore improbable that reading skills were shaped directly by Darwinian selection.

Nevertheless, it is well established that a person's genetic make-up influences their ability to acquire reading and spelling skills, in a manner that can be independent of general cognitive performance. Compelling evidence comes from studying dyslexia, a common neurodevelopmental syndrome involving unexplained reading and spelling difficulties that occur despite normal intelligence and sufficient educational

Corresponding author: Fisher, SimonE(simon.fisher@well.ox.ac.uk).

Available online 3 May 2006 opportunity [2,3]. It has long been known that a family history of dyslexia confers an increased risk of developing reading problems [4]. Twin-based studies indicate the importance of genetic factors [5]. During the past decade, advances in human molecular genetics have allowed researchers to track down chromosomal sites that might harbour factors involved in dyslexia predisposition (reviewed in [6-9]). This work recently turned a major corner, with a rapid succession of discoveries that appear to implicate specific genes in abnormal reading development [10-16].

If we can uncover genetic mechanisms that contribute to dyslexia susceptibility, this will greatly inform our understanding of the cognitive architecture underlying both disrupted and normal reading [8]. In addition to its importance for dyslexia diagnosis and therapy, success in this area will address how specific genetic variants relate to variability in different cognitive skills - such as phonologic awareness and orthographic coding - in the wider population [17]. At a more fundamental level, genes offer a molecular window into the human brain [18], promising to shed light on how the relevant neural circuits function, how they are established and maintained, what their origins are (developmental and evolutionary), and why they yield uneven cognitive profiles when they go awry.

In this article, we focus on four prominent candidate genes from the current literature: DYX1C1 [10], KIA00319 [11-13], DCDC2 [14,15] and ROBO1 [16], and consider whether we are finally in a position to describe genetic mechanisms that underlie dyslexia. We aim to provide guidance for those who are unfamiliar with molecular genetics, allowing the non-specialist to evaluate better how recent genetic discoveries might help explain cognition. It is first essential to discard naïve models in which genomes are perceived as static blueprints, and genes as abstract entities with an enigmatic capacity for directly controlling cognition. Rather, through cascading changes of gene expression in time and space, a genome directs the self-assembly of a complex multicellular organism containing a highly organized central nervous system (CNS), and endows that organism with the ability to respond dynamically to its environment. As we will illustrate, this area of research can be understood only in light of a deeper appreciation of genes, genomes and brains [17]. 


\section{Clues from chromosome anomalies: discovering DYX1C1}

For over two decades, geneticists have searched for correlations between genetic variability and susceptibility to dyslexia (Box 1). The first report of a possible link [19] involved variation around the centromere of chromosome 15 , which was however unsubstantiated in later investigations [20,21]. Nevertheless, other chromosome-15 regions have been implicated repeatedly in reading and spelling disabilities, particularly 15q15-21, now referred to as the DYX1 (dyslexia-susceptibility-1) locus [22-28]. (During searches for genetic risk factors, a chromosomal interval that has been highlighted by linkage/association studies can be assigned an official locus symbol by the international Gene Nomenclature Committee, even before a specific gene has been implicated.) It has proved difficult to determine a precise location; whereas some studies highlight 15q15.1 [26,27,29], others point to markers in 15q21, mapping at least 8 million nucleotides away $[24,25,28]$.

This kind of uncertainty - a pervasive problem when studying genetically complex traits - can sometimes be overcome by the serendipitous discovery of affected individuals who carry gross chromosomal rearrangements involving the relevant genomic region. Nopola-Hemmi and colleagues identified a Finnish family in which a translocation involving chromosomes 15 and 2 (Figure 1) was co-inherited with reading and writing difficulties in a father and three of his four children [30]. The chromosome-15 breakpoint lay within $15 q 21$, disrupting a gene

\section{Box 1. Connecting genes with cognition}

On average, if you line up your DNA with that of an unrelated neighbour, $\sim 3$ million nucleotide letters (approximately $0.1 \%$ of your genome) will not match. Most differences are functionally silent, but others alter protein structure or regulation of gene expression in a way that leads to individual variability in appearance, metabolism, behaviour, disease susceptibility, and so on. When a nucleotide mismatch is present at an appreciable frequency in a population, it is called a single nucleotide polymorphism (SNP). Other types of genetic polymorphism include short tandemlyrepeated sequences (STRs), for which the number of copies differs from person to person. By looking for genotype-phenotype correlations in human populations it is possible to uncover connections between genes and aspects of human biology that are difficult or impossible to explore in animal models.

Linkage analysis uses genetic polymorphisms to track inheritance of different chromosomal regions within families. This technique can provide approximate genomic location(s) of variants influencing a trait, and is particularly suitable for genome-wide screening $[43,50-$ 52]. Association analysis searches on a finer genomic scale for population-wide correlations between a phenotype (e.g. dyslexia diagnosis) and specific allelic variants [59]. A case-control design is often used, in which frequencies of different genotypes are compared between affected and unaffected individuals. Alternatively, a 'transmission disequilibrium' approach asks whether one allelic variant is passed on to affected children more often than others. Because specific allelic variants in adjacent genetic markers tend to be inherited together they are sometimes analyzed together in combinations that are known as 'haplotypes'. Linkage and association methods have also been adapted for analyzing quantitative indices of severity (e.g. performance on reading-related tasks) [8]. Complex genetic traits involve multiple factors with potentially subtle effects, so large samples (perhaps thousands of individuals) can be required to pinpoint the relevant genes.

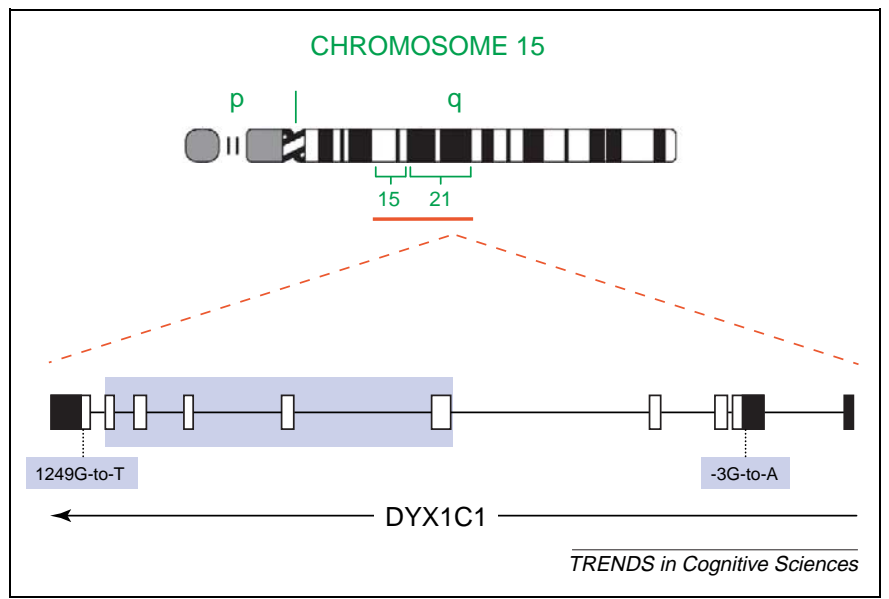

Figure 1. The $D Y X 1 C 1$ gene. An ideogram of chromosome 15 is shown at the top, illustrating the normal banding pattern seen in cytogenetic studies. Human chromosomes contain a short (' $p$ ') arm and a long $\left({ }^{\prime} q\right.$ ') arm, separated by a structure called a centromere. The red bar shows the $D Y X 1$ region, identified by linkage and association studies of dyslexia, corresponding to cytogenetic bands 15q15-21. The bottom half of the figure shows the genomic organisation of the $D Y X 1 C 1$ gene (previously known as EKN1), spanning $\sim 78$ thousand nucleotides in 15q21. Boxes indicate exons (included in the mature RNA transcript), lines indicate introns (removed by splicing), and the arrow shows the direction of transcription. (Note that exons and introns are not shown to the same scale here; some of the introns are very large.) Black shading indicates exons that are not translated into protein sequences (non-coding exons) upstream and downstream of the proteincoding region. Taipale and colleagues [10] studied a Finnish family in which dyslexia was associated with a gross chromosomal rearrangement known as a translocation; one end of chromosome 15 had been exchanged with part of chromosome 2 in the affected individuals. The site of breakage lies somewhere within the purple-shaded region of the $D Y X 1 C 1$ gene. Taipale et al. identified -3Gto-A and 1249G-to-T SNPs in other Finnish families with dyslexia [10], but further studies demonstrated that these are unlikely to represent functional risk alleles [3136]. (Ideograms are adapted from http://www.pathology.washington.edu/research/ cytopages/idiograms/human/)

that was given the name $D Y X 1 C 1$ (dyslexia-susceptibility1, candidate-1) [10]. The protein encoded by $D Y X 1 C 1$ is found in diverse tissues, including a minority of cortical neurons and glia. It contains three tetratricopeptide repeat domains (motifs that mediate protein-protein interactions), one of which is disrupted by the breakpoint in the translocation family.

Chromosomal rearrangements are often benign; their presence can be coincidental and unconnected with disorder. For the 15q21 translocation family, affected individuals carry one damaged copy and one intact copy of DYX1C1 [10], and the latter might be sufficient to preserve normal function. However, the observation that DYX1C1 disruption co-segregates with dyslexia in four family members increases confidence that it is responsible for the disorder. An aetiological mechanism has not as yet been established. The translocation could yield reduced amounts of functional protein, or generate a shorter product that interferes with normal protein, or even create a novel form of the protein with a damaging effect.

\section{Is the DYX1C1 gene implicated in common cases of dyslexia?}

Taipale and colleagues analysed DYX1C1 sequence variation in the wider Finnish population. Two rare changes, -3G-to-A and 1249G-to-T (Figure 1) were present in dyslexia cases and unaffected controls, but appeared to be more frequent in the cases [10]. Some individuals carried a chromosome harbouring both $-3 \mathrm{~A}$ and $1249 \mathrm{~T}$ 
(a combination of alleles referred to as the 'risk haplotype'); this haplotype was transmitted to affected children more often than expected (transmission disequilibrium, Box 1) [10]. Intriguingly, the two SNPs might potentially affect DYX1C1 function in different ways; -3G-to-A lies in a regulatory region which could modulate levels of DYX1C1 expression (how much gene product is made in a cell), whereas 1249G-to-T creates an early stop codon in the coding region, yielding a shorter DYX1C1 protein.

However, six independent follow-up studies of DYX1C1 did not support the claim that the above alleles predispose to reading problems [31-36]. In total, these investigations analysed more than 1024 individuals or families affected with dyslexia in the UK, US, Canada and Italy [31-36]. The $-3 \mathrm{~A}$ and $1249 \mathrm{~T}$ alleles, although present in all populations, were not associated with reading-related deficits. One investigation proposed that the opposite alleles (-3G/1249G rather than -3A/1249T) showed association with reading-related deficits in Canadian families [31]. The title of this report professed support for the Finnish findings, but the text acknowledged the crucial allelic differences, noting that it is unlikely that these specific DNA changes are contributing to the phenotype' [31].

Could the lack of replication in European, Canadian and American families mean that $-3 \mathrm{~A}$ and $1249 \mathrm{~T}$ alleles increase susceptibility to dyslexia only in the Finnish population? Finland shows greater genetic homogeneity than other populations [37], but it is unlikely that the genetic background is distinctive enough to render Finnish people uniquely susceptible to functional effects of these alleles. A more parsimonious alternative is that $3 \mathrm{~A}$ and $1249 \mathrm{~T}$ alleles are not directly relevant to dyslexia in any population [31-36]. For example, the 1249T truncation might have little consequence for DYX1C function, as it shortens the 420 amino acid protein by only four residues.

There are a number of explanations for inconsistencies between the Finnish study and attempted replications. Observed associations could point to undiscovered aetiological variants in DYX1C1 or in another nearby gene, which (owing to their close proximity) have tended to be inherited together with the -3G-to-A and 1249G-to-T variants. If such causative changes arose on a different genetic background in Finland, this might account for the unique Finnish pattern of association. Alternatively, it has been suggested - based on methodological issues - that the Finnish associations could represent false positives $[32,33,36]$. For example, Taipale et al. incorporated multiple family members into a case-control design without adjusting for relatedness, a practice which introduces biases in allele-frequency estimates. Moreover, their separate observations of transmission disequilibrium exploited information from just a handful of individuals [10]. It has been noted that DYX1C1 maps some distance from the regions of strongest linkage or association in earlier DYX1 studies [32,33,36]. Thus, researchers continue to search $15 \mathrm{q} 15$-21 for genes that influence dyslexia susceptibility [29].

\section{A tale of two genes: KIAA0319 and DCDC2 on chromo- some 6}

Linkage to the short arm of chromosome 6 [23,38,39] represents one of the most well replicated findings in the dyslexia genetics literature [24,40-45]. Linkage/association studies converged on part of $6 \mathrm{p} 23-21.3$, named the DYX2 (dyslexia-susceptibility-2) locus, but the region of consensus contained hundreds of genes. Based on multivariate linkage analyses of quantitative data, DYX2 appears primarily to influence trait variability that is shared between reading-related measures, but is independent of variation in general intelligence (IQ) [46]. By removing reading-related variance that was correlated with IQ, the candidate interval was greatly reduced [11]. In addition, researchers focused attention on more severely affected family members, as DYX2 effects seem to be strongest in these individuals $[11,47]$.

In the past year, reports have proposed that the search for the relevant susceptibility gene might now be over, fuelling excitement in the wider field, accompanied by media speculation that genetic diagnosis is on the horizon. Enthusiasm should be tempered by two important caveats: functional risk alleles have not been defined, and there is actually a profound lack of agreement over the identity of the gene. Although some studies point to a gene known as KIAA0319 [11-13], others favour another nearby gene, $D C D C 2[14,15]$.

\section{The KIAA0319 story}

Francks and colleagues identified a $6 \mathrm{p} 22$ region where multiple SNPs showed replicated association with reading-related measures in large numbers of dyslexia families from Berkshire (UK) and from Colorado (US) [11]. One particular combination of alleles was associated with deficits in both the Berkshire and Colorado datasets (a concordant risk haplotype) and risk-associated variants were at elevated frequency in the most severely affected individuals. The region of interest contained three genes (Figure 2). THEM2 encodes a metabolic enzyme, whereas TTRAP encodes part of a complex pathway supporting fundamental cellular processes, including programmed cell death and immune responses, and both genes are widely expressed. In contrast, KIAA0319 is expressed primarily in nervous tissue [48], is up-regulated in regions of the developing and adult mammalian brain [13,14], and encodes a protein that appears to function at the cellsurface, regulating interactions and adhesion between adjacent neurons $[11,13]$.

Cope et al. similarly reported association in the KIAA0319-TTRAP-THEM2 cluster in an independent case-control sample from Cardiff (UK), which they confirmed via transmission disequilibrium testing [12]. In their study, two KIAA0319 SNPs appeared to associate best with dyslexia. One SNP replaces an alanine with a threonine in the KIAA0319 product, but this change, common in unaffected people, was not unique to risk haplotypes associated with dyslexia [11,12]. Overall, it is unlikely that changes to protein structure are relevant here; instead, the aetiological pathway probably involves altered regulation. Recent functional data indicate that chromosomes that carry a putative risk haplotype give 


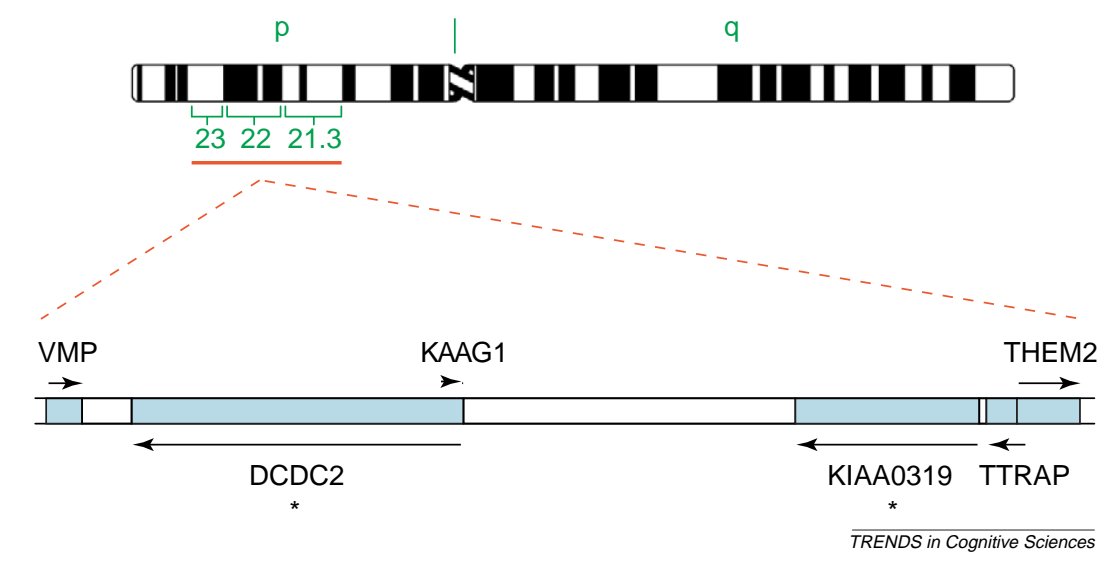

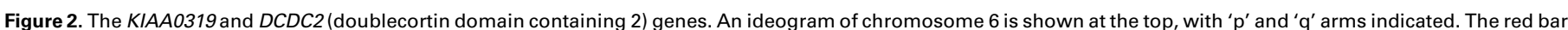

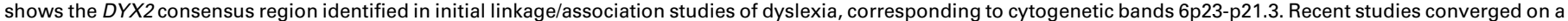

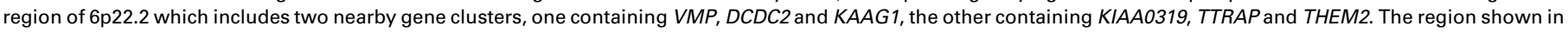

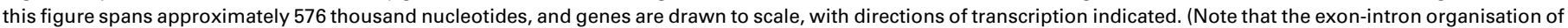

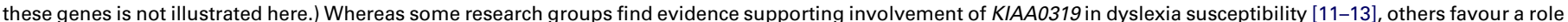
for $D C D C 2[14,15]$

reduced expression of KIAA0319 (but normal levels for THEM2/TTRAP), as compared with chromosomes carrying alternative haplotypes [13]. It is not known which of the sequence variants that are located on this risk haplotype confers this regulatory difference.

\section{The DCDC2 story}

Meng and colleagues investigated the same set of Colorado families as those included in the Francks et al. KIAA0319 study, but homed in on a different gene [14]. In earlier studies, this research group reported positive associations for JA04, a polymorphic marker within KIAA0319 [44]. However, their follow-up work identified stronger associations for SNPs mapping in DCDC2 (Figure 2) [14]. In addition, an intronic region of nearly 2500 nucleotides was missing from $D C D C 2$ in a small number of people. The deletion removes a 168-bp stretch containing STRs (Box 1). When present, these STRs vary in copy number in different people, yielding many different alleles. There was little evidence of association when analysing individual STR alleles or the rare deletion alone, but significant results could be obtained by grouping the deletion with all the low-frequency STR alleles [14]. DCDC2 is expressed in a wide range of tissues, including the brain [15]. Its product shows similarities to DCX, a cytoplasmic protein that has been implicated in neuronal migration deficits in two severe brain disorders; lissencephaly and double cortex syndrome [49].

In a study of German probands with dyslexia, Schumacher et al. independently implicated $D C D C 2$, based on transmission disequilibrium results [15]. Association was most significant for severely affected individuals, but no functional risk alleles were identified, and the Meng et al. deletion/STR was not studied. Like KIAA0319, the functionally relevant sequence changes probably involve regulation of $D C D C 2$ and remain to be discovered. Meng et al. proposed a direct functional role for the deletion/STR polymorphism, based on the computer-based prediction that the STR is a potential target for brain- related regulatory proteins [14]. However, no functional experiments were carried out to assess whether such proteins do indeed bind the STR, and it has not been determined whether STR variation/deletion is associated with changes in DCDC2 expression.

\section{KIAA0319, DCDC2 or both?}

At present, a similarly convincing aetiological argument can be built for either KIAA0319 or DCDC2. For each gene positive association was independently reported in more than one study. Both genes have neural functions that are compatible with the dyslexia phenotype; by reducing expression in utero in rat brain, it has been shown that neuronal migration is impaired by interfering with either KIAA0319 [13] or DCDC2 [14]. In adult brains, each gene is expressed in, but not specific to, broad areas of cerebral cortex which include regions known to be active during fluent reading. Although these data are encouraging, they do not prove a causal link with reading disability. As noted above, a new study has demonstrated that the KIAA0319 risk haplotype is associated with reduced expression of that gene in cultured cells [13]. Still, there has not yet been a direct demonstration for either KIAA0319 or $D C D C 2$ that gene function is disrupted or modified in people with dyslexia. Given the genetic complexity of dyslexia, it is plausible that both genes contribute to risk, but that their relative influence varies in different study samples, and/or depending on analytical approaches.

\section{Altered regulation of the $R O B O 1$ gene in a family with dyslexia}

Linkage to the DYX5 (dyslexia-susceptibility-5) region of chromosome 3 was found in one unusual four-generation Finnish family, in which 27 of 74 members were diagnosed with dyslexia [50]. Reading disability usually shows complex inheritance [8], but in this family transmission was more straightforward, consistent with damage to a single gene, acting in a dominant fashion. (Other reports of apparent simple inheritance include a Norwegian 


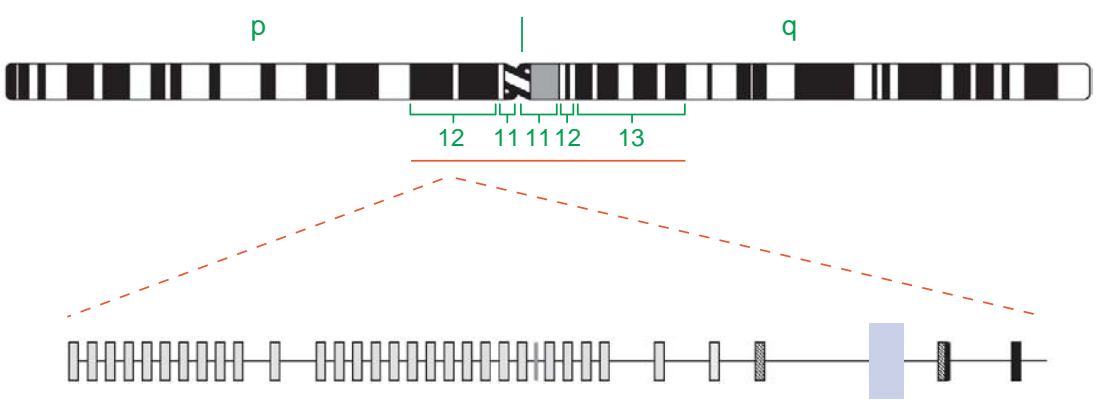

ROBO1/DUTT1

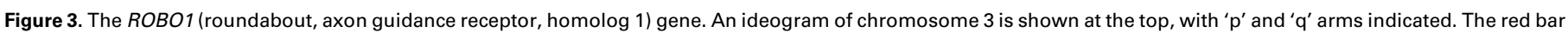

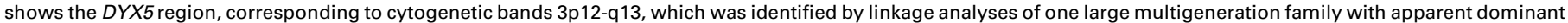

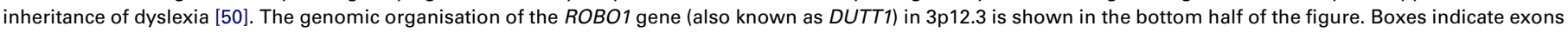

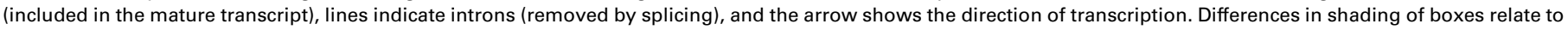

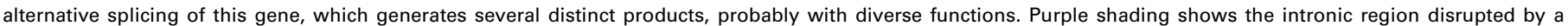
translocation breakpoint in an individual with dyslexia (unrelated to the large DYX5-linked family) [16]. Part of this figure is adapted from [16].

family showing chromosome-2 linkage [51] and a Dutch family showing chromosome-X linkage [52].) As illustrated by investigations of monogenic speech disorder [53-55], studying such families can help identify susceptibility genes. Even if these same genes are not disrupted in common forms of disorder, they can deliver relevant neurogenetic insights [56,57].

One section of chromosome 3 (3p12-q13) was shared by descent (inherited from the same founder chromosome) in 19 of 21 affected individuals studied in the DYX5-linked family [50]. Helpful clues came from discovery of an unrelated individual with dyslexia who carried a gross chromosomal anomaly - a translocation involving chromosomes 3 and 8 [16]. Hannula-Jouppi and colleagues discovered that the chromosome-3 breakpoint in this independent case disrupted an intron of $R O B O 1$ (Figure 3). Robo, the fruitfly version of $R O B O 1$, encodes a transmembrane receptor involved in signal transduction, which helps regulate axon/dendrite guidance. Robo mutations cause abnormalities in the ways that axons cross the midline of the fruitfly CNS [58].

$R O B O 1$ maps in $3 \mathrm{p} 12$, in the region that is identical-bydescent in the majority of affected people from the large DYX5-linked family. On screening the gene in this family, it was discovered that affected people carried an unusual combination of SNP alleles. One identified variant affects ROBO1 protein structure by inserting/deleting an aspartic-acid residue, but is also found frequently in unaffected controls and unlikely to be causal [16]. Hannula-Jouppi et al. hypothesized that the $R O B O 1$ risk haplotype in the family harboured an undetected regulatory mutation. Therefore, they investigated levels of gene expression in lymphocytes from four affected family members, finding that $R O B O 1$ expression from the risk haplotype was lower than that from its 'normal' counterpart. (Each affected individual carries two copies of chromosome 3, only one of which harbours the risk haplotype.)

These data suggest that disrupted expression of one chromosomal copy of $R O B O 1$ can predispose to dyslexia, perhaps owing to reduced ROBO1 protein in the CNS.
However, there are several complications. The child with the chromosome-3 translocation has a sister who also has severe dyslexia, but does not carry the translocation. With regard to the DYX5-linked family, it is unknown whether the lymphocyte expression findings are representative of the situation in brain tissue. Moreover, it has not been shown that reduced ROBO1 expression is correlated with dyslexia in each of the 19 members carrying the risk haplotype, only in pooled data from four affected individuals. 3p12-q13 contains many genes that have never been examined in the DYX5-linked family, so it remains possible that their disorder results from an alternative aetiological change in an unstudied gene.

\section{Uniting themes}

Dyslexia research has entered a new phase in which positive genetic associations are being reported. Genetic association indicates a statistical correlation between carrying an allelic variant and manifesting a trait [59], but does not demonstrate causality. Complex traits are notorious for providing false positive (and negative) results; hence the need for unambiguous independent replication [60]. Even replicated association can be misleading, given that neighbouring SNPs tend to be coinherited. Positive association might reflect functional effects of changes elsewhere in the same genomic region, perhaps in a different gene. Thus, distinguishing between correlation and cause is a central issue for the field [17,60].

Extensive screening of protein-coding regions has failed to find structural changes that are implicated in dyslexia susceptibility. Coding changes that have been found (the DYX1C1 truncation [10], the KIAA0319 alanine-to-threonine substitution $[11,12]$ and the ROBO1 aspartic-acid deletion/insertion [16]) appear to have little functional relevance. Therefore, any aetiological variants within these candidate genes probably involve altered regulation, in line with a widespread recognition that regulatory changes make major contributions to complex human traits [61]. 
It could be difficult to identify the crucial regulatory changes. Because genetic association cannot distinguish between a truly functional SNP and neighbouring variants 'hitchhiking' along with it, the burden of proof lies with other approaches. Indirect evidence might come from model systems (like genetically manipulated rodents) or comparing expression levels of candidates in different regions of the human brain [14]. Indeed, it can be valuable to characterize the normal pattern of human CNS expression for a gene that has been implicated in a neurodevelopmental disorder, suggesting mechanisms by which gene disruption leads to impairment [62].

However, if a gene is normally expressed in adult cortical regions known to be active during reading, this does not itself constitute evidence of a pathological link between that gene and dyslexia. Gene expression profiles show considerable overlap for different regions of adult human cortex, each of which contains a highly complicated mixture of cell-types [63]. Moreover, adult patterns do not necessarily reflect developmental expression, and genes like DCDC2/KIAA0319 have been proposed to influence dyslexia through developmental mechanisms (abnormal neuronal migration) [14], rather than altered 'online' roles in reading-related circuits in the mature brain. Definitive evidence that an allelic variant is

\section{Box 2. Reading, genes and evolution}

The existence of genes that influence reading ability appears at odds with the recent cultural emergence of written language. However, although variability in a gene might yield increased susceptibility to reading disability, this does not mean that the gene evolved in order to provide humans with reading skills. Genes implicated in dyslexia need not be unique to our species; rodents carry versions of the $D Y X 1 C 1, K I A A 0319, D C D C 2$ and $R O B O 1$ genes that are very similar to those in humans.

Nevertheless, reading processes might recruit neural substrates that evolved in support of human language, and it is generally accepted that dyslexia often involves subtle disruption of linguistic skills $[2,3]$. It is therefore of interest to examine how candidate genes changed during primate evolution [18]. Altered rates of protein evolution can be detected by comparing protein-coding sequences in different species. It is not sufficient simply to quantify inter-species amino-acid differences, because background mutation rates can differ hugely from one genomic region to another. Instead, the number of nucleotide changes that alter amino-acids should be compared with the number of 'silent' changes that preserve protein sequence.

Some analyses of evolutionary changes in dyslexia candidate genes have failed to make this correction for local mutation rate. For example, Taipale et al. proposed accelerated change of DYX1C1 in recent primate evolution because three out of 420 amino-acids differ between humans and chimpanzees [10]. In fact, this does not exceed the number expected from the local mutation rate, because the coding region was also reported to harbour four silent differences, suggesting that $\mathrm{DYX} 1 \mathrm{C} 1$ has not changed more rapidly than expected. Similarly, it has been proposed that ROBO1 underwent accelerated protein change on lineages leading to humans, chimpanzees and gorillas, as compared with the lineage leading to the orang-utan [16]. However, for each lineage there is a large excess of silent changes, and $R O B O 1$ actually represents one of the more slowly evolving genes of the genome. (See ref. [18] for further discussion of primate comparative genomics, language evolution and examples of robust accelerated change). Evolutionary histories of $K I A A 0319$ and $D C D C 2$ have yet to be investigated. Thus, it remains to be seen whether dyslexia will be informative for studies of human evolution. aetiological requires a demonstration that it leads to alterations in regulation/function of the gene in question, and that these alterations are present in people with dyslexia.

Lacking essential functional data, we should be cautious before offering genetic diagnoses using the currently known SNPs, STRs, and deletions. Levels of association reported so far, which result from global analyses of large samples, are not strong enough to translate into reliable predictors of risk for a single individual.

\section{Conclusion: reading the genome}

None of the candidate genes discussed here could accurately be described as a 'gene for reading' - that is, a gene whose primary function is to support reading acquisition and/or performance. Individual genes do not specify behavioural outputs or cognitive skills, or even particular neural circuits. Genes influence brain development and function interactively by affecting processes such as proliferation and migration of neurons, programmed cell-death, axonal pathfinding, connectivity, levels of neurotransmitters/receptors, and so on. $D Y X 1 C 1, K I A A 0319$ and $D C D C 2$ are implicated in neuronal migration $[13,14,64]$, and $R O B O 1$ might affect axon crossing [58] and cortical dendrite development [65], but these roles are not specific to reading-related circuitry. For example, $D C D C 2$ is expressed in many regions of adult human brain [15], peaking in sub-cortical structures (amygdala, hippocampus and hypothalamus) [14].

In addition, expression of these candidate genes is not confined to the CNS; DYX1C1 and DCDC2 show high

\section{Box 3. Questions for future research}

- What are the specific functional variants in candidate genes such as KIAAO319 and DCDC2 that predispose an individual to dyslexia?

- Why should alteration of a gene that is normally expressed in a wide range of brain structures lead to selective cognitive deficits, rather than global impairment?

- Do susceptibility genes affect cognitive phenotypes via disruptions to embryonic development or altered 'online' function during cognitive processing (or both)?

- How does genetic variability relate to variations in cognitive profile in people with dyslexia? Some genetic studies of dyslexia fractionate the overall phenotype into hypothetical 'component' phenotypes, such as phoneme awareness, rapid automized naming and orthographic coding. Early suggestions that different genes might have specific separable effects on these processes have not held up, but it remains plausible that risk alleles impact more strongly on certain phenotypic aspects than on others.

- What are the longitudinal effects on cognitive profile for people carrying particular risk alleles?

- Can we correlate genetic information with data from structural or functional neuroimaging, for example to discover whether people who carry putative risk alleles show common anomalies in brain development/function?

- Does genetic profile influence the response of an individual to particular behavioural interventions, and will we ever be able to target therapies based on an individual's genetic information?

- Can genetic information account for high comorbidities between dyslexia and other neurodevelopmental disorders, such as specific language impairment and attention-deficit/hyperactivity disorder? Are there 'shared' genetic risk factors that increase susceptibility to multiple disorders? 
levels in lung, kidney and testis [10,15]. Mammalian $R O B O 1$, which is assembled into several forms via a process called alternative splicing, is implicated in mammalian cancers [66,67]. Moreover, reading is a human-specific skill, but each candidate gene is present in a similar form in diverse species (Box 2). Such findings therefore fit with an emerging picture in which human cognitive traits involve recruitment and refinement of evolutionarily ancient mechanisms [18].

If it is confirmed that one or more of these candidate genes influences dyslexia susceptibility, this will aid the development and differentiation of effective behavioural interventions for remediation of reading problems. It is hoped that further studies will help to explain why aetiological variants disturb reading while generally preserving broader aspects of cognition (see Box 3). Intriguingly, altered regulation of genes involved in neuronal migration and/or connectivity is concordant with neurobiological data emerging from other avenues of research $[3,64,68]$. It is already clear that synergy between molecular approaches and distinct forms of enquiry, like neuroimaging and behavioural studies, will be powerful both for improving prospects of treating dyslexia and for uncovering neural pathways that contribute to reading.

\section{References}

1 Lawler, A. (2001) Archaeology. Writing gets a rewrite. Science 292, 2418-2420

2 Vellutino, F.R. et al. (2004) Specific reading disability (dyslexia): what have we learned in the past four decades? J. Child Psychol. Psychiatry $45,2-40$

3 Demonet, J.F. et al. (2004) Developmental dyslexia. Lancet 363, $1451-1460$

4 Hallgren, B. (1950) Specific dyslexia (congenital word-blindness); a clinical and genetic study. Acta Neurol. Scand. Suppl. 65, 1-287

5 Gayán, J. and Olson, R.K. (2001) Genetic and environmental influences on orthographic and phonological skills in children with reading disabilities. Dev. Neuropsychol. 20, 483-507

6 Grigorenko, E.L. (2001) Developmental dyslexia: an update on genes, brains, and environments. J. Child Psychol. Psychiatry 42, 91-125

7 Schulte-Körne, G. (2001) Annotation: genetics of reading and spelling disorder. J. Child Psychol. Psychiatry 42, 985-997

8 Fisher, S.E. and DeFries, J.C. (2002) Developmental dyslexia: genetic dissection of a complex cognitive trait. Nat. Rev. Neurosci. 3, 767-780

9 Francks, C. et al. (2002) The genetic basis of dyslexia. Lancet Neurol. $1,483-490$

10 Taipale, M. et al. (2003) A candidate gene for developmental dyslexia encodes a nuclear tetratricopeptide repeat domain protein dynamically regulated in brain. Proc. Natl. Acad. Sci. U. S. A. 100, 11553-11558

11 Francks, C. et al. (2004) A 77-kilobase region of chromosome 6p22.2 is associated with dyslexia in families from the United Kingdom and from the United States. Am. J. Hum. Genet. 75, 1046-1058

12 Cope, N. et al. (2005) Strong evidence that KIAA0319 on chromosome $6 \mathrm{p}$ is a susceptibility gene for developmental dyslexia. Am. J. Hum. Genet. 76, 581-591

13 Paracchini, S. et al. (2006) The chromosome 6p22 haplotype associated with dyslexia reduces the expression of KIAA0319, a novel gene involved in neuronal migration. Hum. Mol. Genet. (Epub ahead of print)

14 Meng, H. et al. (2005) DCDC2 is associated with reading disability and modulates neuronal development in the brain. Proc. Natl. Acad. Sci. U. S. A. $102,17053-17058$

15 Schumacher, J. et al. (2005) Strong genetic evidence of DCDC2 as a susceptibility gene for dyslexia. Am. J. Hum. Genet. 78, 52-62
16 Hannula-Jouppi, K. et al. (2005) The axon guidance receptor gene ROBO1 is a candidate gene for developmental dyslexia. PLoS Genet. 1, e50

17 Fisher, S.E. Tangled webs: tracing the connections between genes and cognition. Cognition (in press)

18 Fisher, S.E. and Marcus, G.F. (2006) The eloquent ape: genes, brains and the evolution of language. Nat. Rev. Genet. 7, 9-20

19 Smith, S.D. et al. (1983) Specific reading disability: identification of an inherited form through linkage analysis. Science 219, 1345-1347

20 Bisgaard, M.L. et al. (1987) Dyslexia and chromosome 15 heteromorphism: negative lod score in a Danish material. Clin. Genet. 32, 118-119

21 Rabin, M. et al. (1993) Suggestive linkage of developmental dyslexia to chromosome 1p34-p36. Lancet 342, 178

22 Fulker, D.W. et al. (1991) Multiple regression of sib-pair data on reading to detect quantitative trait loci. Reading and Writing 3, 299-313

23 Smith, S.D. et al. (1991) Screening for multiple genes influencing dyslexia. Reading and Writing 3, 285-298

24 Grigorenko, E.L. et al. (1997) Susceptibility loci for distinct components of developmental dyslexia on chromosomes 6 and 15. Am. J. Hum. Genet. 60, 27-39

25 Schulte-Körne, G. et al. (1998) Evidence for linkage of spelling disability to chromosome 15. Am. J. Hum. Genet. 63, 279-282

26 Morris, D.W. et al. (2000) Family-based association mapping provides evidence for a gene for reading disability on chromosome 15q. Hum. Mol. Genet. 9, 843-848

27 Marino, C. et al. (2004) A locus on 15q15-15qter influences dyslexia: further support from a transmission/disequilibrium study in an Italian speaking population. J. Med. Genet. 41, 42-46

28 Chapman, N.H. et al. (2004) Linkage analyses of four regions previously implicated in dyslexia: confirmation of a locus on chromosome 15q. Am. J. Med. Genet. B. Neuropsychiatr. Genet. 131, $67-75$

29 Morris, D.W. et al. (2004) Association analysis of two candidate phospholipase genes that map to the chromosome 15q15.1-15.3 region associated with reading disability. Am. J. Med. Genet. B. Neuropsychiatr. Genet. 129, 97-103

30 Nopola-Hemmi, J. et al. (2000) Two translocations of chromosome 15q associated with dyslexia. J. Med. Genet. 37, 771-775

31 Wigg, K.G. et al. (2004) Support for EKN1 as the susceptibility locus for dyslexia on 15q21. Mol. Psychiatry 9, 1111-1121

32 Scerri, T.S. et al. (2004) Putative functional alleles of DYX1C1 are not associated with dyslexia susceptibility in a large sample of sibling pairs from the UK. J. Med. Genet. 41, 853-857

33 Cope, N.A. et al. (2005) No support for association between dyslexia susceptibility 1 candidate 1 and developmental dyslexia. Mol. Psychiatry 10, 237-238

34 Marino, C. et al. (2005) A family-based association study does not support DYX1C1 on $15 \mathrm{q} 21.3$ as a candidate gene in developmental dyslexia. Eur. J. Hum. Genet. 13, 491-499

35 Bellini, G. et al. (2005) No Evidence for Association Between Dyslexia and DYX1C1 Functional Variants in a Group of Children and Adolescents From Southern Italy. J. Mol. Neurosci. 27, 311-314

36 Meng, H. et al. (2005) TDT-association analysis of EKN1 and dyslexia in a Colorado twin cohort. Hum. Genet. 118, 87-90

37 Varilo, T. and Peltonen, L. (2004) Isolates and their potential use in complex gene mapping efforts. Curr. Opin. Genet. Dev. 14, $316-323$

38 Cardon, L.R. et al. (1994) Quantitative trait locus for reading disability on chromosome 6 . Science $266,276-279$

39 Cardon, L.R. et al. (1995) Quantitative trait locus for reading disability: correction. Science 268,1553

40 Fisher, S.E. et al. (1999) A quantitative-trait locus on chromosome 6p influences different aspects of developmental dyslexia. Am. J. Hum. Genet. 64, 146-156

41 Gayán, J. et al. (1999) Quantitative-trait locus for specific language and reading deficits on chromosome 6p. Am. J. Hum. Genet. 64, 157-164

42 Grigorenko, E.L. et al. (2000) Chromosome 6p influences on different dyslexia-related cognitive processes: further confirmation. Am. J. Hum. Genet. 66, 715-723 
43 Fisher, S.E. et al. (2002) Independent genome-wide scans identify a chromosome 18 quantitative-trait locus influencing dyslexia. Nat. Genet. 30, 86-91

44 Kaplan, D.E. et al. (2002) Evidence for linkage and association with reading disability on 6p21.3-22. Am. J. Hum. Genet. 70, 1287-1298

45 Turic, D. et al. (2003) Linkage disequilibrium mapping provides further evidence of a gene for reading disability on chromosome 6p21.3-22. Mol. Psychiatry 8, 176-185

46 Marlow, A.J. et al. (2003) Use of multivariate linkage analysis for dissection of a complex cognitive trait. Am. J. Hum. Genet. 72, 561-570

47 Deffenbacher, K.E. et al. (2004) Refinement of the 6p21.3 quantitative trait locus influencing dyslexia: linkage and association analyses. Hum. Genet. 115, 128-138

48 Londin, E.R. et al. (2003) A transcription map of the $6 \mathrm{p} 22.3$ reading disability locus identifying candidate genes. BMC Genomics 4, 25

49 Gleeson, J.G. et al. (1998) Doublecortin, a brain-specific gene mutated in human X-linked lissencephaly and double cortex syndrome, encodes a putative signaling protein. Cell 92, 63-72

50 Nopola-Hemmi, J. et al. (2001) A dominant gene for developmental dyslexia on chromosome 3. J. Med. Genet. 38, 658-664

51 Fagerheim, T. et al. (1999) A new gene (DYX3) for dyslexia is located on chromosome 2. J. Med. Genet. 36, 664-669

52 de Kovel, C.G. et al. (2004) Genomewide scan identifies susceptibility locus for dyslexia on Xq27 in an extended Dutch family. J. Med. Genet. $41,652-657$

53 Fisher, S.E. et al. (1998) Localisation of a gene implicated in a severe speech and language disorder. Nat. Genet. 18, 168-170

54 Lai, C.S. et al. (2001) A forkhead-domain gene is mutated in a severe speech and language disorder. Nature 413, 519-523

55 MacDermot, K.D. et al. (2005) Identification of FOXP2 truncation as a novel cause of developmental speech and language deficits. Am. J. Hum. Genet. 76, 1074-1080
56 Fisher, S.E. et al. (2003) Deciphering the genetic basis of speech and language disorders. Annu. Rev. Neurosci. 26, 57-80

57 Marcus, G.F. and Fisher, S.E. (2003) FOXP2 in focus: what can genes tell us about speech and language? Trends Cogn. Sci. 7, 257-262

58 Kidd, T. et al. (1998) Roundabout controls axon crossing of the CNS midline and defines a novel subfamily of evolutionarily conserved guidance receptors. Cell 92, 205-215

59 Cardon, L.R. and Bell, J.I. (2001) Association study designs for complex diseases. Nat. Rev. Genet. 2, 91-99

60 Page, G.P. et al. (2003) 'Are we there yet?' Deciding when one has demonstrated specific genetic causation in complex diseases and quantitative traits. Am. J. Hum. Genet. 73, 711-719

61 Knight, J.C. (2005) Regulatory polymorphisms underlying complex disease traits. J. Mol. Med. 83, 97-109

62 Lai, C.S. et al. (2003) FOXP2 expression during brain development coincides with adult sites of pathology in a severe speech and language disorder. Brain 126, 2455-2462

63 Khaitovich, P. et al. (2004) Regional patterns of gene expression in human and chimpanzee brains. Genome Res. 14, 1462-1473

64 Ramus, F. (2004) Neurobiology of dyslexia: a reinterpretation of the data. Trends Neurosci. 27, 720-726

65 Whitford, K.L. et al. (2002) Regulation of cortical dendrite development by Slit-Robo interactions. Neuron 33, 47-61

66 Dallol, A. et al. (2002) Tumour specific promoter region methylation of the human homologue of the Drosophila Roundabout gene DUTT1 (ROBO1) in human cancers. Oncogene 21, 3020-3028

67 Xian, J. et al. (2004) Targeted disruption of the $3 \mathrm{p} 12$ gene, Dutt1/Robo1, predisposes mice to lung adenocarcinomas and lymphomas with methylation of the gene promoter. Cancer Res. 64, 6432-6437

68 Galaburda, A.M. et al. (1985) Developmental dyslexia: four consecutive patients with cortical anomalies. Ann. Neurol. 18, 222-233

\section{Elsevier.com - Dynamic New Site Links Scientists to New Research \& Thinking}

Elsevier.com has had a makeover, inside and out. Designed for scientists' information needs, the new site, launched in January, is powered by the latest technology with customer-focused navigation and an intuitive architecture for an improved user experience and greater productivity.

Elsevier.com's easy-to-use navigational tools and structure connect scientists with vital information - all from one entry point. Users can perform rapid and precise searches with our advanced search functionality, using the FAST technology of Scirus.com, the free science search engine. For example, users can define their searches by any number of criteria to pinpoint information and resources. Search by a specific author or editor, book publication date, subject area - life sciences, health sciences, physical sciences and social sciences - or by product type. Elsevier's portfolio includes more than 1800 Elsevier journals, 2200 new books per year, and a range of innovative electronic products. In addition, tailored content for authors, editors and librarians provides up-to-the-minute news, updates on functionality and new products, e-alerts and services, as well as relevant events.

Elsevier is proud to be a partner with the scientific and medical community. Find out more about who we are in the About section: our mission and values and how we support the STM community worldwide through partnerships with libraries and other publishers, and grant awards from The Elsevier Foundation.

As a world-leading publisher of scientific, technical and health information, Elsevier is dedicated to linking researchers and professionals to the best thinking in their fields. We offer the widest and deepest coverage in a range of media types to enhance crosspollination of information, breakthroughs in research and discovery, and the sharing and preservation of knowledge. Visit us at Elsevier.com.

Elsevier. Building Insights. Breaking Boundaries. 